\title{
Analysing Enterprise Models from a Fractal Organisation Perspective - Potentials and Limitations
}

\author{
Kurt Sandkuhl $^{1}$ and Marite Kirikova ${ }^{2}$ \\ ${ }^{1}$ University of Rostock, Department of Computer Science \\ Albert-Einstein-Str. 22, 18059 Rostock, Germany \\ Kurt. Sandkuhl@uni-rostock. de \\ ${ }^{2}$ Riga University of Technology, \\ Kalku 1, Riga, LV-1658, Latvia \\ Marite.Kirikovalcs.rtu.1v
}

\begin{abstract}
The paper focuses on the use of the fractal paradigm in enterprise modeling. It investigates whether the properties of fractal organizations can be applied in business analysis and whether this results in useful outcomes and new insights. Based on an adaptation and operationalization of properties of fractal organizations, two real-world cases are analyzed using the adapted properties. The contributions of this paper are (1) to adapt fractal organisation properties for use in analysis of enterprise models, (2) to present practical examples from two cases showing the application of the fractal organisation properties, and (3) to identify potentials and limitations of using fractal organisation perspective in enterprise model analysis.
\end{abstract}

Keywords: Enterprise Modelling, Fractal Organisation, Practices, Business Analysis.

\section{Introduction}

Work presented in this paper brings together approaches and experiences from two different research fields in computer science; enterprise modelling and fractal organisations. In enterprise modelling, one of the traditional application purposes has been to understand the current situation in an enterprise or organisation under consideration in order to identify or explain business problems and to propose improvements [1]. Many methods, approaches, tools and work practices aiming at supporting this purpose were developed in areas such as business process reengineering [2], process improvement [3], enterprise knowledge modelling [4] organisational renewal [5], or and information systems development [4]. This large body of knowledge forms one basis for the work presented in this paper. In this context, our focus is on business analysis, i.e. on analysing enterprise models to identify organisational improvement potential.

High turbulence of business environment requires from organizations such features as agility [11], and viability [12]. Both of these features require means for achieving a good balance between complexity and simplicity in organizational management and operations. Fractal organizational structure is proposed by several researches, e.g. [6], [12], and [13] as an enabler of agility and vitality. 
Fractal organisation structures have received much attention in areas like manufacturing industries or enterprise engineering. Among the advantages of fractals, their flexibility, robustness and easy adaptation to new business challenges are considered as interesting for many application domains. Does it make sense to apply fractal organisation principles when analysing businesses i.e. is it feasible to do this and does it give useful results pertinent to analysis purpose? What are the potential benefits and limitations of doing this?

The contribution of this paper is (1) to adapt fractal organisation properties for use in analysis of enterprise models, (2) to present practical examples from industrial cases showing the pertinence of the fractal organisation properties, and (3) to identify potentials and limitations of using the fractal organisation perspective in enterprise model analysis.

The paper is structured as follows: Section 2 will introduce the fractal paradigm as the background for our research. Section 3 investigates how the properties of fractal organisations can be interpreted in analysis of enterprise models, i.e. an adaptation of the properties of fractal organisation for enterprise modelling is proposed. In Section 4 , we use the adapted properties proposed in section 3 for analysing real-world enterprise models in order to illustrate pertinence. Section 5 discusses the results of applying the fractal organisation perspective and identifies potentials and limitations of using this perspective. Section 6 summarizes the work and gives an outlook on future work.

\section{$2 \quad$ Background of Fractal Paradigm}

The start of the use of fractal paradigm in context of organizational structure and behaviour can be traced back to 1992 [13]. The overall number of research papers available on this topic is not very large, the number of books is 2 [12], [13]. In the first edition of [13] H. J. Warnecke suggests fractal organization of enterprise as essential means for survival in turbulent environment. In this work a fractal is defined as independently acting corporate entity whose goals and performance can be precisely described. H. J. Warnecke defines and describes the following basic properties of fractal organization:

- Self-similarity - fractals are self-similar, each performs services;

- Self-organization - fractals practice self organizations (1) operatively - procedures are optimally organized by applying suitable methods and (2) tactically and strategically - fractals determine and formulate their goals in dynamic process and decide upon internal and external contacts. Fractals restructure, regenerate and dissolve themselves.

- Goal orientation - the system of goals that arises from the goals of individual fractals is free from contradictions and must serve the objective of achieving corporate goals.

- Dynamics (in other sources named as dynamics and vitality [10]) - (1) the fractals are networked via an efficient information and communication system. They themselves determine the nature and extent of their access to data; (2) the performance of a fractal is subject to constant assessment and evaluation. 
P. Hoverstadt's book [12], written 16 years later than [13] applies the fractal paradigm as the basis for use Viable Systems Model [14] in enterprise management.

In different researches dated from 1993 to 2011 the fractal paradigm has been applied in the following contexts:

- Manufacturing/product development [19], [20], [25], [26]

- Organizational networks [21], [22], [23], [26], [31]

- Service oriented systems [15], [30], [32]

- Agent oriented systems [28], [33]

- Business process and workflow management [25]

- Competence, responsibility, motivation, and goal management [16], [17], [18], [24], [27], [31]

- Knowledge and decision making management [21], [24]

- Quality control [19], [28]

- Enterprise architecture [29]

- Information systems and software engineering ( a survey of related work in information systems development is included in [34]), [35]

The above list of topics shows that many organizational aspects are researched from the point of view of the fractal paradigm; however there is no analysis on applicability of fractal paradigm in enterprise modelling practice. Usefulness of the paradigm in each of the above mentioned enterprise perspectives suggests that use of the paradigm could bring particular benefits in enterprise modelling. The fractal paradigm does not focus on isolated aspects of an organisation, but defines properties cross-cutting processes, organisation structures and information flows, to name just a few examples. Such a multi-perspective approach including different aspects is also essential for enterprise modelling, since in enterprise modelling it is important to understand dependencies and connections between different organisational aspects (e.g. processes, organisation structure, competences or enterprise architecture), because organisational changes or problems usually affect several aspects.

\section{Fractal Organisation Properties for Enterprise Model Analysis}

Based on the properties and characteristics of fractal organisations described in section 2, this section investigates the adaptation of these properties for use in business analysis. The assumption made for work presented in this paper is that the "as is" situation in an enterprise already has been captured and documented in a model, i.e. we focus on analysis of models rather than on developing them. In this context, adaptation of properties includes two aspects:

- How to interpret the property in the context of business analysis?

- How to operationalize this interpretation for practical use, i.e. what questions to investigate in an actual analysis case and how to perform the analysis?

In the remaining part of this section, we will discuss the properties of fractal organisations from section 2 with their interpretation in business analysis and the operationalization. The focus will be on self-similarity, goal orientation, selforganisation, and dynamics. 


\section{Self-similarity}

As discussed in section 2, self-similarity is a repetition of a particular pattern of organization structure at different scales of a particular organizational dimension or at different scales of several organizational dimensions simultaneously. If such a pattern exists in some organization unit of the same scale, but not in all units of this scale, the reason for this should be investigated. Are pattern-compatible units performing better or worse than non-pattern units? Should there be an adaptation towards the pattern of the non-pattern structure?

Based on the above interpretation of the property, the proposed operationalization includes the following questions to be investigated during business analysis:

SS-1: Do organisation patterns repeat on different scales of the organisation in the dimension "organisation structure"? If so, does the repeating pattern has advantages compared to other structures and should be implemented on all scales?

SS-2, SS-3, and SS-4: Same as SS-1, but for product structure (SS-2), process structure (SS-3), resources structure ( $S S-4)$.

This property also includes that information should no longer be monopolized, but be made generally available. In practice support for this aspect has to be established in the enterprise architecture and implemented by information systems in the enterprise. More obvious solutions would include access to essential information from all organisational functions. Enterprise models include relations from functions to information systems, i.e. we could use these relations as indicators.

Operationalization (continued):

SS-5: Is the information system structure included in the model? If so, do all organisation units have access to essential information systems?

\section{Self-organisation}

Self-organisation includes that fractals restructure, regenerate and dissolve themselves based on goals in a dynamic process and internal and external contacts. If the model of the enterprise under consideration indicates delegation of decision rights regarding enterprise strategy implementation to organization units, these units should have continuous improvement and adaptation processes in place and it also has to include adaptation. Furthermore, there should be organizational roles responsible for this task.

\section{Operationalization:}

SO-1: Is delegation of responsibilities in the organization reflected in the model? If so, do continuous improvement and adaptation processes exist?

SO-2: Have organizational roles responsible for continuous improvement been established?

In the literature on fractal organisation, so called "process patterns" characterizing fractal organisations are described. An example is the pattern described by [10], which identifies monitoring, analyzing, reporting, planning, executing as the main functions in project-oriented fractal companies. Such process patterns for fractal organisations could serve as templates for analysing models in order to detect selforganisation structures. 


\section{Operationalization (continued):}

SO-3: Can all processes from the process pattern be found in the enterprise model under consideration? If so, can additional properties of fractal organisations be identified?

SO-4: Can the majority of the sub-processes of the process pattern be found in the model? If so, are the missing processes - if not named in a different way - the starting point for improving the organisation into this direction?

\section{Goal Orientation}

Goal orientation includes different aspects, like that the goals of individual fractals are free of contradictions from the goals of the overall organization, serve the objective of achieving corporate goals and involve all units concerned. Goal orientation as a property does not need adaptation for use in business analysis, since even non-fractal organisations should follow this principle. However, to determine whether this property is implemented is not a trivial task if only the enterprise models are available. It would basically require propagation of the goals to different organisational division and the refinement of relevant goals for each organisational unit

\section{Operationalization:}

GO-1: Are the enterprise's goals included in the enterprise model? If so, are the goals broken down for use in different organisational units? Should this be done in order to reach acceptance, as it is recommended in a balanced scorecard with its subscorecards?

\section{Dynamics and Vitality}

The operationalization of this property overlaps with SO-1, SO-2, SS-5, and GO1. Additionally fractals with identical goals and input and output variables can have quite different internal structures. If organization units with identical input/outputs and goals exist, is one of the internal structures superior to the others and should it be adapted by the others?

\section{Operationalization:}

DV-1: Do different processes, activities, or tasks with identical input/output variables exist in the model? If so then if they have different internal structures, is there a superior one performing better?

The above operationalization of self-similarity, goal orientation, self-organisation, and dynamics focuses on the questions to ask in business analysis. Furthermore, we also need to define, how to use these questions in the actual business analysis. As discussed in Section 3, we assume that an enterprise model exists capturing the "as is" situation. Although there are different ways how to use the questions for analysing an enterprise model, we recommend the following procedure and use it in the cases presented in Section 4: The business analysis should be performed by a team consisting of at least one expert in fractal organisation properties familiar with the questions and one enterprise modelling expert familiar with the model under consideration. The analysis should start with an introduction of scope and purpose of the model, the method and notation used, and a walk-through of the actual model, which starts with an overview 
to the main processes and structures, and includes at least one part of the model in full detail. Afterwards, the questions are analysed as follows:

- SS-1 to SS-4: the analysis team jointly browses different perspectives (process, organization, product, resources) of the enterprise model on different levels. Similarities are documented as pattern candidates. After finishing the browsing, all candidates are revisited in order to decide whether they are patterns.

- SO-1, SO-2 and GO-1: the analysis team checks the meta-model for entity types or relationships types matching the wanted ones (i.e. role, delegation, goal). For the matching entity types, the instances are browsed to answer the questions.

- SO-3 and SO-4: the analysis team browses the instances of the process-related entity-types in the model for instance names matching the names of the subprocesses in the process patterns.

- DV-1: same procedure as for SS-1 to SS-4, but limited to those parts of the model containing processes, activities or tasks.

\section{Application of the Adapted Properties in Real-World Cases}

This section focuses on evaluating the adapted properties introduced in section 3 regarding the applicability and pertinence by applying them in two cases. One case is taken from the public sector and the other one from industry. Section 4.1 presents and discusses the application of the adapted property in the public authority case; section 4.2 covers the industrial case. An interpretation of the results and comparison of the cases is included in section 5 .

\subsection{Public Authority Case}

The Public Authority case model was developed in 2005. Its purpose was to establish a vision of new information systems of a university. Currently the information system has been developed. Its functionality is taken into consideration in the enterprise model analysis. The enterprise model was developed using Enterprise Knowledge Development methodology [7]. The model consists of five sub-models reflecting perspectives of Goals, Processes, Actors and Resources, Concepts, and Information Systems Components and Requirements.

The results of analysis of the model are presented according to the four properties of fractal organizations and their operationalization introduced in Section 3.

\section{Self-similarity}

SS-1: Do organisation patterns repeat on different scales of the organisation in the dimension "organisation structure"? If so, does the repeating pattern has advantages compared to other structures and should it be implemented on all scales?

The pattern at different scales was identified in Actors and Resources sub-model. It was Unit level $i$, Management of Unit level $i$, binary relationship between Unit level $i$ and Management of Unit level $i$. The levels in decreasing order of granularity were University, Faculty, Institute, and Department. A representative of University IT department participating in the model analysis session admitted that clear repetition of 
the same pattern at all scales would give an opportunity to state clearer and richer information systems requirements.

SS-2: Do structural patterns repeat on different scales of the organisation in the dimension "product structure"?

Product structure was not represented in the model.

SS-3: Do process patterns repeat on different scales of the organisation in the dimension "process structure"? If so, does the repeating pattern has advantages compared to other structures and should it be implemented on all scales?

In the business process model which was developed at a high level of abstraction common patterns where not found except of some similarities with respect to maintenance of different Information systems registers related to each level of organizational structure. Relationships between the registers were not specified.

SS-4: Do structure patterns repeat on different scales of the organisation in the dimension "resource structure"?

Resources (excluding organizational structure) were represented at a high level of detail and did not contain repeating patterns.

SS-5: Is the information system structure included in the model? If so, do all organisation units have access to essential information systems?

Information systems structure was partly represented in Concepts sub-model. Indirect relationships to all organizational units were identified in the model.

\section{Self-organisation}

SO-1: Is delegation of responsibilities in the organization reflected in the model?

Model showed only which information is received or transferred by which actor. Delegation of responsibilities was not represented.

SO-2: Have organizational roles responsible for continuous improvement been established?

The activity of continuous improvement was identified in the model, but was not assigned to any actor. This can be explained with the fact that in 2005 there was no specific department responsible for continuous improvement. Some years later a University Strategy Department was established which partly deals with issues of continuous improvement. An interesting fact is that this department has its own information system that operates on different principles than other parts of the information system and causes lots of problems for users due to request of inputs that do not fit the work structure. The Strategy Department's system was outsourced from another public institution. The difficulties currently perceived emphasize that it is necessary to represent who and how is responsible for continuous improvement in the enterprise model to avoid problems that can arise if this issue is not considered.

SO-3: Can all processes from the process pattern be found in the enterprise model under consideration?

Planning process was missing.

SO-4: Can the majority of the sub-processes of the process pattern be found in the model? 
Processes of the process pattern (except of planning) were represented in the model, however they did not form fractal structure.

\section{Goal Orientation}

GO-1: Are the enterprise's goals included in the enterprise model? If so, are the goals broken down for use in different organisational units? Should this be done in order to reach acceptance?

The models included a sub-model dedicated to the goals of the university; however, the goals were not broken down for use in different organizational units. Particular goals for units at all levels would be beneficial so as to achieve clearer and richer requirements and reach user acceptance for information systems solutions.

\section{Dynamics and Vitality}

DV-1: Do different processes, activities, or tasks with identical input/output variables exist in the model? If so, then if they have different internal structures, is there a superior one performing better?

On the highest level of abstraction it was possible to identify such inputs and outputs for processes related to different registers. These processes were not shown in detail and thus it was not possible to analyze their internal structure. In another project related to university information system [35] such structures were identified and recommended for implemention at several branches of the fractal organization.

\subsection{Industrial Case}

The industrial model analyzed was developed in the EU-FP6-project MAPPER [9] during 2006 - 2008. The model was produced in the automotive supplier use case of MAPPER and focused on the department of advanced engineering and the 'process of innovation', which basically involves several organizational functions in developing new technologies or components for future products of the company. The method used was C3S3P [5, 6]. Two model versions were available: the model of the 'as is' situation, also called scoping model, and the model slowing the 'to be' situation, also called solution model.

The models covered the POPS* perspectives [8], which include the enterprise's processes $(\mathrm{P})$, the organization structure $(\mathrm{O})$, the product developed $(\mathrm{P})$, the IT system used $(\mathrm{S})$ and other aspects deemed relevant when modeling (*). The analysis of the models from MAPPER is presented in the following and divided according to the four properties and their operationalization introduced in Section 3.

\section{Self-similarity}

SS-1: Do organisation patterns repeat on different scales of the organisation in the dimension "organisation structure"?

Solution model and scoping model included only the advanced engineering department in full detail. Other organisation structures were not included. Thus, similarity between organisation units could not be detected. 
SS-2: Do structural patterns repeat on different scales of the organisation in the dimension "product structure"?

The scoping did not contain any product structure, but it was decided to include the product decomposition structure, requirements, technical characteristics, materials, etc. in the solutions model. Structural similarities between different levels of the product structure were detected, but these similarities were based only on common characteristics of different component types. Further unification of the structures on different levels wouldn't make sense since the different kinds of product components (sensors, harness, wires, etc.) have significantly different structures and features.

SS-3: Do process patterns repeat on different scales of the organisation in the dimension "process structure"? If so, does the repeating pattern has advantages compared to other structures and should it be implemented on all scales?

Regarding the work processes, the scoping model did not contain any similarities between different levels, which were not surprising since the processes were only very roughly defined without details and refinements. In the solution model, similarity exists on two levels. First, all tasks aiming at "establishing" a specification start with "preparing" the task and continue by "developing an initial draft", "establishing test methods" and the "final specification". The second similarity was detected on a refinement level. Wherever a material, a test method or a design approach had to be selected, first the existing ones were checked for suitability, then the decision was made whether to develop a new one in-house or to outsource this.

SS-4: Do structure patterns repeat on different scales of the organisation in the dimension "resource structure"?

The only resources included in the scoping model were the major IT systems used in the enterprise. This was primarily to identify the systems and did not show their internal structure or relationships. The solution model included more structure and refinements of this aspect, but similarity on different scales was not detected.

SS-5: Is the information system structure included in the model? If so, do all organisation units have access to essential information systems?

To improve and promote access to information and knowledge, and sharing of such information was one of the main intentions of the project. Thus, the solution model contains many details of which view of what information is needed for what role in what task. All roles and all organisation units have access to essential information. This property of a fractal organisation is clearly visible in the model.

\section{Self-organisation}

SO-1: Is delegation of responsibilities in the organization reflected in the model? and SO-2: Have organizational roles responsible for continuous improvement been established?

Mechanisms and structures for continuous improvement and adaptation of the organisation structure were outside the scope of the modelling. The model focused on the core process "Process of Innovation" of one organisation unit "advanced engineering" but did not cover the whole organisation unit or the whole organisation. 
SO-3: Can all processes from the process pattern be found in the enterprise model under consideration? and SO-4: Can the majority of the sub-processes of the process pattern be found in the model?

Such structures are not part of the model. The model did not cover the whole organisation, nor the whole organisation unit. Whether the process pattern exists in the organisation or not cannot be concluded from the model.

\section{Goal Orientation}

GO-1: Are the enterprise's goals included in the enterprise model? If so, are the goals broken down for use in different organisational units? Should this be done in order to reach acceptance?

The models included a sub-model dedicated to the goals of the MAPPER project and the goals of the company for the POI. These goals were captured as goal hierarchy, the goals were linked to processes and organizational units related to goal fulfillment.

This goal orientation and the traceability of the goals were implemented in the solution model since a number of specific goals regarding knowledge sharing and innovation were very important for the company and should be addressed. In the scoping model, goals were not included and the specific goals not defined. Thus, the case is a good example for the importance of goal orientation in industry and a confirmation of the relevance of this property of fractal organizations.

\section{Dynamics and Vitality}

DV-1: Do different processes, activities, or tasks with identical input/output variables exist in the model? If so, then if they have different internal structures, is there a superior one performing better?

On a high aggregation level, many processes exist with identical input and output, since most processes of the POI have the product knowledge as input and the changed product knowledge as output. However, on a lower level, these identical inputs are no longer visible, since only different part of the product knowledge is used.

\section{Discussion}

This section is dedicated to discussing the experience acquired in the two cases presented in section 4 . The approach used for this discussion is to compare the results of analysing the enterprise models of the two cases with the results of the original business analysis performed in these cases, i.e., the analysis results of the enterprise models developed in these real-world cases are considered as "gold standard" when comparing the results of the analysis with the adapted properties. For both cases, a table will be presented summarizing two aspects of the evaluation:

- Applicability: Was it possible to apply the property and its operationalization in practice?

- Usefulness: Was the use of the properties and its operationalization of any help in business analysis? 
Regarding applicability four categories will be distinguished:

- it was possible to apply the operationalization and the property was detected

- it was possible to apply the operationalization, but the property was not detected

- It remains unclear, whether the operationalization can be applied (e.g. if the model does not contain information needed for applying the property)

- it was not feasible to apply the operationalization Regarding usefulness, five categories will be distinguished:

- New insights: using the property gave new insights and other opportunities to improve the organization as compared to the result of the original business analysis

- Confirmed: using the property resulted in confirmation of the results of the original business analysis, i.e. the same or compatible results were achieved

- Contradiction: using the property resulted in recommendations contradicting the results of the original business analysis.

- Not relevant: the property was not relevant for the business analysis

- Not applicable: the property could not be analyzed, e.g. due to missing data Table 1 shows a summary of the analysis of the public authority case.

Table 1. Summary of public authority case

\begin{tabular}{|c|c|c|}
\hline Property & Applicable? & Usefulness \\
\hline$S S-1$ & Property detected & $\begin{array}{l}\text { New insights (how to organize work at lower levels } \\
\text { of institutional granularity) }\end{array}$ \\
\hline$S S-2$ & Property not detected & $\begin{array}{l}\text { Not applicable (property not detected, however the } \\
\text { product perspective could be useful and give new } \\
\text { insights with respect to information systems } \\
\text { requirements) }\end{array}$ \\
\hline$S S-3$ & $\begin{array}{l}\text { Property detected (to } \\
\text { some extent) }\end{array}$ & $\begin{array}{l}\text { New insights (more detailed analysis of these } \\
\text { properties could help to identify particular } \\
\text { requirements at different levels of fractal } \\
\text { organization) }\end{array}$ \\
\hline$S S-4$ & Property not detected & Not applicable (property not detected) \\
\hline$S S-5$ & Property detected & $\begin{array}{l}\text { New insights (direct instead of indirect relationships } \\
\text { could give better chances for interface requirements } \\
\text { definition) }\end{array}$ \\
\hline$S O-1$ & Not detected & $\begin{array}{l}\text { Not applicable (aspect missing in the model except } \\
\text { of information flows to and from actors) }\end{array}$ \\
\hline$S O-2$ & $\begin{array}{l}\text { Property detected (to } \\
\text { some extent) }\end{array}$ & $\begin{array}{l}\text { There is a process for continuous improvement but } \\
\text { there is no delegation: New insight: modelling the } \\
\text { delegation is essential in case of continuous } \\
\text { improvement. }\end{array}$ \\
\hline $\mathrm{SO}-3$ & Not detected & Not applicable (property not detected) \\
\hline $\mathrm{SO}-4$ & Property detected & $\begin{array}{l}\text { New insight (related to } \mathrm{SO}-2 \text {; it is essential to } \\
\text { represent planning process in an enterprise model }\end{array}$ \\
\hline$G O-1$ & $\begin{array}{l}\text { Property detected (to } \\
\text { some extent) }\end{array}$ & $\begin{array}{l}\text { New insight: it is beneficial to propagate goals in } \\
\text { organisation structure }\end{array}$ \\
\hline$D V-1$ & $\begin{array}{l}\text { Property detected (to } \\
\text { some extent) }\end{array}$ & $\begin{array}{l}\text { Confirmation: in another research of university } \\
\text { information system preferable process structures } \\
\text { were found. }\end{array}$ \\
\hline
\end{tabular}


This summary shows that it was not possible to detect fractal properties $S S 2, S S 4$, $S O 1$, and SO3. Some properties, namely, SS3, SO2, GO1, and DV1, were detected to some extent. Nevertheless, the results of analysis of fractal properties showed that utilization of them in enterprise models could provide richer models and more detailed information systems requirements.

Table 2. Summary of industrial case

\begin{tabular}{|l|l|l|}
\hline Property & Applicable? & Usefulness \\
\hline$S S-1$ & Property not detected & Not applicable (property not detected) \\
\hline$S S-2$ & Property detected & $\begin{array}{l}\text { Confirmation and contradiction: some part of the } \\
\text { structures should repeat, but unification of product } \\
\text { structures wouldn't make sense. }\end{array}$ \\
\hline$S S-3$ & Property detected & Confirmation: processes with similar structure \\
\hline$S S-4$ & Property not detected & Not applicable (property not detected) \\
\hline$S S-5$ & Property detected & Not applicable (property not detected) \\
\hline $\begin{array}{l}S O-1 \\
S O-4\end{array}$ to & unclear & Not applicable (aspect missing in the model) \\
\hline$G O-1$ & Property detected & $\begin{array}{l}\text { Confirmation: goals have to be propagated in } \\
\text { organisation structure }\end{array}$ \\
\hline$D A-1$ & Property detected & Not applicable (aspect missing in the model) \\
\hline
\end{tabular}

Table 2 shows a summary of the analysis of the industrial case. This summary shows that it was possible to apply the operationalization of the properties in the industrial case. Only the properties for self-organization could not be utilized since the scope of the model was too narrow as it was limited to one department and one value creation process. Furthermore, most of the other properties were not detected in the model, indicating that the enterprise under investigation does not show many characteristics of a fractal organization.

However, those properties which were detected confirmed the results of the original business analysis, i.e. utilization of self-similarity for the process and product perspective (SS-2 and SS-3) and goal orientation (GO-1) are relevant and valuable for the enterprise under consideration. Self-Similarity in the product perspective also showed a case where it is important to see and respect limitations of self-similarity, since some levels of the product structure have similarities, but these similarities disappear with increasing specialization.

The presented research focused on a most common canonical list of the properties of fractal systems, namely, self-similarity at different levels of scale, self organization, goal-orientation, and dynamics and vitality [36]. In [37] there are other properties of fractal systems listed, such as emergence, co-evolution, sub-optimality, requisite variety, connectivity, and simple rules. Operationalization of these properties may generate some new questions besides the ones described in Section 3. However, further research is needed to see to what extent the analysis of these additional properties is possible and useful in the context of enterprise modelling.

\section{Conclusions and Future Work}

The aim of the paper was to investigate whether the properties of fractal organizations can be applied in business analysis and whether this results in useful outcomes and 
new insights. Based on an adaptation and operationalization of properties of fractal organizations, two real-world cases were analyzed using the adapted properties. The first case showed many properties of fractal organizations and led to new insights regarding further improvement potential of the organization. In the second case, the results were not as positive as in the first case, but still showed the applicability of the properties and importance of properties, like goal orientation and self-similarity.

Although the use of fractal organization properties in business analysis showed some promising results, the potential would probably be bigger if we started to use them already when capturing the "as is" situation in an enterprise. This could help to avoid certain shortcomings in analysis models, like missing delegation relations between roles or improvement processes, but the methods for enterprise modeling might have to be adapted. An example is the property of self-similarity of the processes in the industrial case: during development of the solution model in this case, the processes were designed separately from each other and only after several iterations of refinement and validation a similarity between them developed. This refinement process probably would have been shorter with the advantages of selfsimilarity in mind. One of the main issues in this context is the method support. Enterprise modeling methods like EKD or C3S3P do not include activities supporting the implementation of certain organization paradigms, like fractal organizations, and probably should not do so, since this would limit the applicability and the situational adaptability of the methods. However, for business analysis and process design activities, it might be beneficial to raise awareness on the analysis side for advantages of fractal organization forms and to offer additional method support, like a method component for fractal organization based process design.

The main limitation of the work is the small number of cases considered in the evaluation and the limited number of fractal organisation properties applied. Basically we can conclude that it is possible to apply our operationalization and that this was contributing to business analysis in these two cases, but we should not even try to generalize these results. Since the results from these first two cases are promising, future work will have to focus on identifying characteristics of cases or organisations, where the use of fractal organisation properties in business analysis can be recommended. More cases are required in order to reach conclusions on this question. Furthermore, the operationalization has to be subject to a more thorough quality check including (a) do we need to include more properties or a different interpretation of fractal organisation properties? and (b) can the operationalization be made more precise, complete and easier to apply? This aspect requires involvement of more practitioners.

Acknowledgment. We acknowledge Ilze Birzniece, Ligita Businska, Janis Drobisevskis, and Peteris Rudzajs for analysis of the of public authority enterprise model. We acknowledge the work in the MAPPER project for developing the industrial model.

\section{References}

1. Harmon, P.: The Scope and Evolution of Business Process Management. In: vom Brocke, J., Rosemann, M. (eds.) Handbook on Business Process Management. Springer, Heidelberg (2009) 
2. Davenport, T.H.: Process Innovation. Reengineering Work Through Information Technology. Harvard Business School Press, Boston (1993)

3. Humphrey, W.S.: Software Process The improvement - A Personal View: How it Started and Where it is Going. Software Process The improvement and Practice 12, 223-227 (2007)

4. Lillehagen, F., Krogstie, J.: Active Knowledge Modeling of Enterprises. Springer, Heidelberg (2008)

5. Warner, B.W.: Organisational Development - A Process of Learning and Changing. Addison Wesley, Reading (1994)

6. Sandkuhl, K., Lillehagen, F.: The Early Phases of Enterprise Knowledge Modeling: Practices and Experiences from Scaffolding and Scoping. In: Stirna, J., Persson, A. (eds.) IFIP PoEM 2008. LNBIP, vol. 15, pp. 1-14. Springer, Heidelberg (2008)

7. Bubenko, jr., J., Persson, A., Stirna, J.: EKD User Guide. Appendix B to Deliverable 3 of EU-funded project "HyperKnowledge" (\#IST-2000-28401) (2001)

8. Lillehagen, F.: The Foundations of AKM Technology. In:10th International Conference on Concurrent Engineering (CE) Conference, Madeira, Portugal (2003)

9. Johnsen, S., Schümmer, T., Haake, J., Pawlak, A., Jørgensen, H., Sandkuhl, K., Stirna, J., Tellioglu, H., Jaccuci, G.: Model-based Adaptive Product and Process Engineering. In: Rabe, M., Mihók, P. (eds.) New Technologies for the Intelligent Design and Operation of Manufacturing Networks. Fraunhofer IRB Verlag, Stuttgart (2007)

10. Canavesio, M.M., Martinez, E.: Enterprise modeling of a project-oriented fractal company for SMEs networking. Computers in Industry 58(8-9), 794-813 (2007)

11. Jordan, J.A.: Requirements for next generation manufacturing systems. In: Proceedings of the IEEE/CPMT International Manufacturing Technology Symposium, pp. 247-255. IEEE, Los Alamitos (1994)

12. Hoverstadt, P.: The Fractal Organization: Creating Sustainable Organization with the Viabable System Model. John Wiley \& Sons, Chichester (2008)

13. Warnecke, H.J.: The Fractal Company: A Revolution in Corporate Culture. Springer, Heidelberg (1993)

14. Beer, S.: Platform for Change. John Wiley, Chichester (1978)

15. Zhao, Y., Wu, J., Shu, H.: The fractal management of SOA based services integration. In: Proceedings of International Conference on Information Management Innovation Management and Industrial Engineering, pp. 420-424. IEEE, Los Alamitos (2008)

16. Deng, X., Peng, J., Huang, H.: Research on the fractal company modelling based competence, pp. 2136-2140. IEEE, Los Alamitos (2009) 978-1-4244-3672-9/09

17. Klopp, M., Kibling, W., Splavack, M.: Fractal lines, Manufacturing and Engineering, pp. 82-85 (April 1997)

18. Li, H., And Li, X.: An analysis of the double module fractal structures of enterprises and its forming motivations and mechanisms. In: Proceedings of International Workshop on Chaos-Fractals Theories and Applications, pp. 422-425. IEEE, Los Alamitos (2009)

19. Qin, Y., Zhao, L., Yao, Y., Xu, D.: A study of inter-enterprise quality control function selforganization reconfiguration based fractal networks. In: Proceedings of the 2007 IEEE International Conference on Robotics and Biomimetics, pp. 1733-1737. IEEE, Los Alamitos (2007)

20. Bin, H., Gangyan, L.: Configuration unit for product self-organizing configuration design and its application. In: Proceedings of Fifth International Conference on Natural Computing, pp. 468-472. IEEE, Los Alamitos (2009)

21. Xu, J., Chen, C.: Similarity in tacit knowledge of supply chain and interfaces, p. 5. IEEE, Los Alamitos (2010) 978-1-4244-7161-4/10 
22. Fan, X., Chen, H.: Research on the self-organization model of fractal supply chain, pp. 14. IEEE, Los Alamitos (2008) 978-1-4244-2108-4/08

23. He, X.: Research on evaluation model for self-similarity of fractal supply chain. In: International Conference on Educational and Information Technology (ICEJT 2010), pp. VI-279-VI-281 (2010)

24. Lei, Z., Shouju, R., Zuzho, L.: Self-organization based DSS framework, pp. 609-614. IEEE, Los Alamitos (2000) 0-7803-6583-6/00

25. Strauss, R.E., Hummel, T.: The new industrial engineering revisited, information technology, business process reengineering, and lean management in self-organizing fractal company. In: Proceedings of 1995 Engineering Management Conference, pp. 287292. IEEE, Los Alamitos (1995)

26. Danli, D., Leng, J., Hongyan, Z.: A ship manufacturing model for fractal-based green supply chain. In: Proceedings of MSIE 2011, pp. 22-25. IEEE, Los Alamitos (2011)

27. Mun, J., Shin, M., Jung, M.: Trust evaluation model for fractal-based virtual enterprises using goal achievement probability. In: Proceedings of the 2007, IEEE IEEM, pp. 15321536. IEEE, Los Alamitos (2007)

28. Xu, D., Zhao, L., Yao, Y.: Fractal and mobile agent-based inter-enterprise quality tracking and control (2008)

29. Malan, R., Bredemeyer, D.: The art of change: Fractal and emergant. Enterprise Architecture 13(5), 1-25 (2010)

30. Traverson, B.: Contract management in viewpoint specifications: Experiment in the Electrical Network and SOA domains. In: Proceedings of the 14th International Enterprise Distributed Object Computing Conference Workshops, pp. 361-367 (2010)

31. Mun, J., Shin, M., Wi, H., Lee, K., Jung, M.: A VO formation process of a project-based organization with trust evaluation using goal achievment probability. In: Proceedings of PICMET 2008, pp. 256-265 (2008)

32. Nayak, N., Nigam, A.: Modeling business services for implementing global business services delivery platforms. In: The Proceedings of the 9th International Conference on ECommerce Technology and The 4th International Conference on Enterprise Computing, ECommerce and E-Sevices (CEC-EEE 2007), 8 p. (2007)

33. Gustavsson, P.M., Planstedt, T.: The road towards multi-hypoyhesis intention simulation agents architecture- Fractal information fusion modeling. In: Kuhl, M.E., Steiger, N.M., Armstrong, F.B., Joines, J.A. (eds.) Proceedings of the 2005 Winter Simulation Confernece, pp. 2532-2541 (2005)

34. Kirikova, M.: Towards multifractal approach in IS development. In: Barry, C., Conboy, K., Lang, M., Wojtkowski, G., Wojtkowski, W. (eds.) Information Systems development: Challenges in Practice, Theory and Education, vol. 1, pp. 295-306. Springer, Heidelberg (2009)

35. Stecjuka, J., Makna, J., Kirikova, M.: Best practices oriented business process operation and design. In: Proceedings of the 9th Workshop on Business Process Modeling, Development and Support Business Process Life-Cycle: Design, Deployment, Operation \& Evaluation Proceedings of the BPMDS 2008 Workshop held in Conjunction with the CAiSE 2008 Conference Montpellier, France, June 16-17, pp. 171-184. CEUR (2008)

36. Ryu, K., Jung, M.: Fractal approach to managing intelligent enterprises. Creating Knowledge Based Organisations. In: Gupta, J.N.D., Sharma, S.K. (eds.), pp. 312-348. Idea Group Publishers, USA (2003)

37. Fryer, P., Ruis, J.: What are fractal systems: A brief description of complex adaptive and emerging systems (2006), http: / / www . fractal .org 